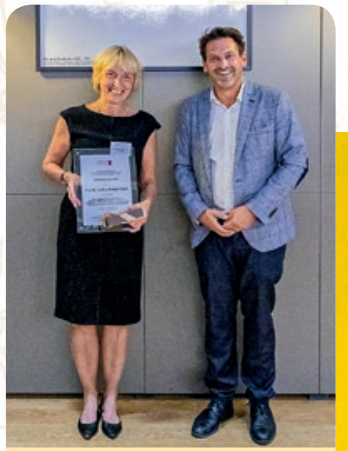

Prof. Dr. med. Andrea Rubbert-Roth und Prof. Dr. med. Thomas Hügle, Präsident des wissenschaftlichen Komitees der SGR (๔ SGR).

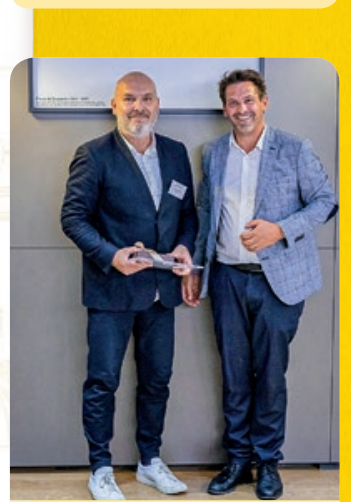

PhD Stavros Giaglis und Prof. Dr. med. Thomas Hügle, Präsident des wissenschaftlichen Komitees der SGR (@ SGR).

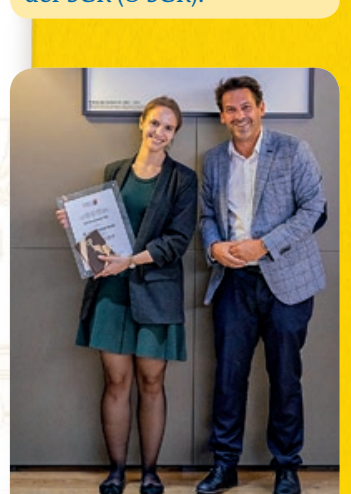

Dr. med. Chantal Meier und Prof. Dr. med. Thomas Hügle, Präsident des wissenschaftlichen Komitees der SGR (๔ SGR).
Kongresspreise der Schweizerischen Gesellschaft für Rheumatologie 2021

Im Rahmen des Jahreskongresses 2021 der Schweizerischen Gesellschaft für Rheumatologie (SGR) hat die SGR die drei am besten bewerteten Abstracts und den besten Case-Report mit dem Kongresspreis 2021 ausgezeichnet. Prof. Dr. med. Andrea Rubbert-Roth vom Kantonsspital St. Gallen erhielt den Preis für das Abstract "Anti-S1 antibodies after vaccination with anti SARS-COV-2 mRNA vaccines in patients with rheumatoid arthritis differ in magnitude and kinetics from healthy controls: Results from a prospective, observational controlled study». PhD Stavros Giaglis von der Universität Basel und vom Universitätsspital Basel wurde für sein Abstract «Plasma Mitochondrial DNA as a Biomarker in the Diagnosis and Follow-up of Systemic Lupus Erythematosus» ausgezeichnet. Dr. med. Chantal Meier vom Universitätsspital Zürich wurde für das Abstract «HRCT-based Radiomics for Prediction of Treatment Response in SScILD» bedacht, und Dr. med. Fabian Lötscher vom Inselspital Bern wurde für den Case-Report «Genetic double strike: VEXAS and TET2 positive myelodysplastic syndrome in a patient with long-standing refractory autoinflammatory disease» ausgezeichnet. Der Preis ist mit je CHF 1000 dotiert.

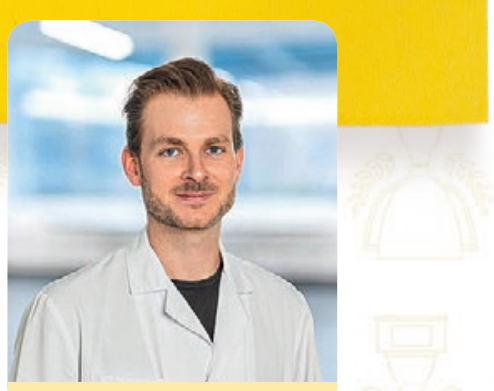

Dr. med. Fabian Lötscher, Inselspital Bern (๑ Pascal Gugler / Insel Gruppe AG).

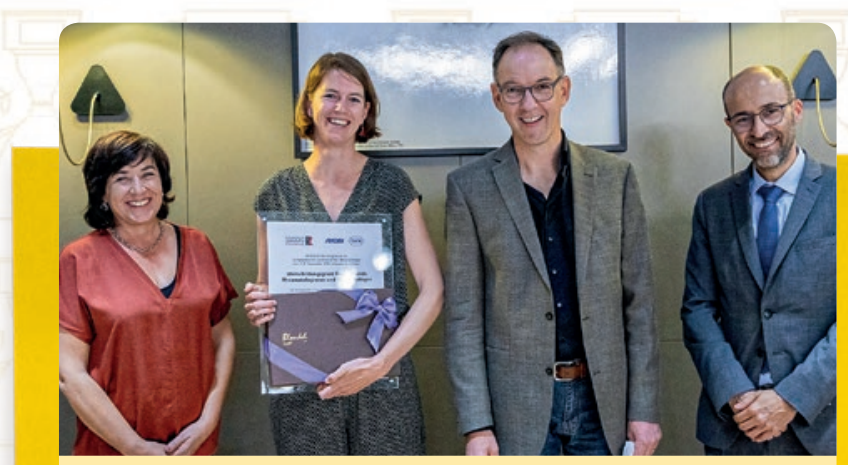

(v.l.n.r.) Cristina Venier, Regional Healthcare Partner, Roche Pharma (Schweiz) AG; Dr. med. Jennifer Amsler, Inselspital Bern; Prof. Dr. med. Daniel Aeberli, Präsident der SGR; Ivan De Martin Del Zotto, Product Manager Inflammation, AMGEN Switzerland AG (๑ SGR).

Weiterbildungs-Grant für angehende Rheumatologinnen und Rheumatologen 2021

Dr. med. Jennifer Amsler vom Inselspital Bern erhält für ihr Forschungsprojekt «Rolle der Neutrophilen in der Entzündung bei Rheumatoider Arthritis und Arthrose» den WeiterbildungsGrant für angehende Rheumatologen 2021. Der mit CHF 50000 dotierte Grant ist dazu bestimmt, angehenden Rheumatologinnen resp. Rheumatologen eine Weiterbildung im Ausland für die Dauer von mindestens sechs Monaten zu ermöglichen. Jennifer Amsler führt ihre Forschungsarbeit an der Universität von Paris durch. Finanziert wird der von der Schweizerischen Gesellschaft für Rheumatologie (SGR) verliehene Grant zur Hälfte von der SGR und je zu einem Viertel von der AMGEN Switzerland AG und der Roche Pharma (Schweiz) AG.

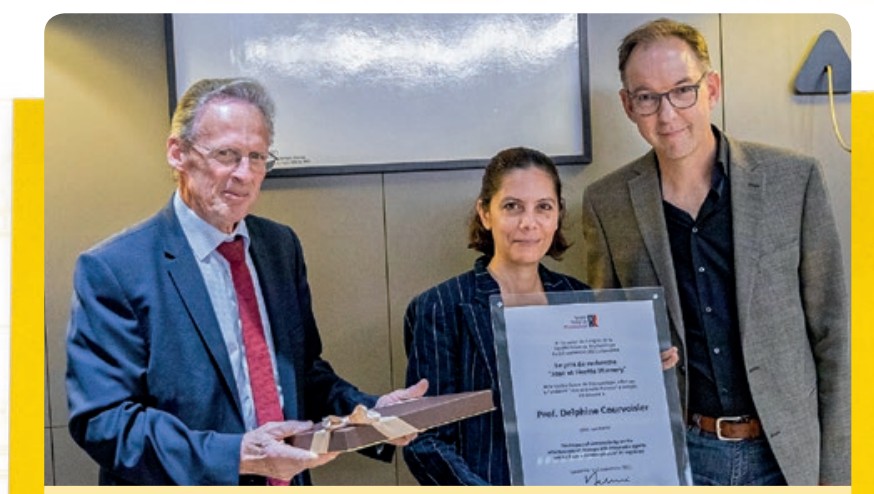

Dr Emile Simon, président de la Fondation Jean et Linette Warnery, Prof. Delphine Courvoisier, HUG, et Prof. Dr méd. Daniel Aeberli, président de la Société Suisse de rhumatologie (๑ SSR).

\section{Prix de recherche Jean et Linette Warnery 2021}

Le prix de recherche en arthrite rhumatoïde récompense un ou plusieurs travaux de recherche clinique ou fondamentale concernant l'arthrite rhumatoïde. Offert par la Fondation Jean et Linette Warnery à Morges, le prix, doté de CHF 60000 , a été décerné par la Société Suisse de Rhumatologie (SSR) à Prof. Delphine Courvoisier, HUG, pour son travail «The impact of seropositivity on the effectiveness of biologic anti-rheumatic agents: results from a collaboration of 16 registries». 